\title{
Priorités de la recherche forestière au Canada pour 1992
}

\author{
Survol à l'intention du Conseil canadien des ministres des forêts rédigé par le \\ Conseil consultatif de la recherche forestière du Canada \\ en collaboration avec les comités consultatifs de la recherche \\ forestière des provinces et des territoires \\ Ottawa, septembre 1992
}

\begin{abstract}
Résumé
Ce quatrième survol annuel des priorités de la recherche forestière pour l'ensemble du Canada a été colligé par le Conseil consultatif de la recherche forestière du Canada pour le Conseil canadien des ministres des forêts. Il se fonde sur les cinq priorités en matière de recherche forestière indiquées par les organismes provinciaux et territoriaux chargés de la question.

Les priorités ainsi signalées ont été cotées en fonction d'une échelle allant de la cote 5 , pour une priorité absolue, à la cote 1, pour l'aspect le moins prioritaire des cinq. La liste de 10 sujets ainsi obtenue est présentée ici, dans l'ordre décroissant de priorité :

- gestion intégrée des ressources et appui à la décision;

- gestion des ravageurs et des mauvaises herbes et solutions de rechange aux produits chimiques;

- effets de l'aménagement forestier sur l'environnement;

- connaissances écologiques pour l'aménagement intensif des forêts;

- données sur l'accroissement et le rendement des forêts;

- accroissement de la productivité, amélioration des arbres et régénération;

- inventaire des forêts et classification des sites;

- méthodes de sylviculture et de récolte et réduction de leurs coûts;

- gestion et répression des incendies de forêt;

- gestion des forêts mixtes.
\end{abstract}

La recherche sur la transformation du bois et sur la création de nouveaux produits est aussi considérée comme importante, mais n'étant pas le but principal du Conseil consultatif de la recherche forestière du Canada, elle n'a donc pas été cotée. Les systèmes modernes de traitement de l'information, l'intelligence artificielle et la biotechnologie figuraient également parmi les points à considérer, mais ce sont des moyens plutôt que des sujets de recherche; ils n'ont pas non plus été cotés.

L'évolution des priorités au cours des quatre années écoulées depuis le premier classement a été marquée d'un changement de méthode dans la présentation des rapports, au bout de deux ans. Il est donc impossible de faire une comparaison rigoureuse, mais on peut tout de même arriver à classer globalement (priorité élevée, moyenne, etc.), pour les premières années, les priorités signalées aux fins du présent rapport.

Aucun changement spectaculaire n'est survenu. La «gestion intégrée des ressources et l'appui à la décision» demeure une priorité absolue. L'ascension aux niveaux 2,3 et 4 de l'échelle de trois sujets liés à l'environnement est significative. Il s'agit de "gestion des ravageurs et des mauvaises herbes et solutions de rechange aux produits chimiques», «effets de l'aménagement forestier sur l'environnement» et "connaissances écologiques pour l'aménagement intensif des forêts». Les "méthodes de sylviculture et de récolte et la réduction de leurs coûts» ont également grimpé, tandis que les «données sur l'accroissement et le rendement des forêts» ainsi que l'«accroissement de la productivité forestière, l'amélioration des arbres et la régénération» ont été déclassés. Les autres sujets n'ont pas bougé de façon notable. Les priorités de la recherche ne semblent pas changer plus rapidement que ne peuvent s'y adapter les organismes de recherche.

Douze facteurs ont été signalés comme pouvant influer sur l'établissement des priorités de la recherche :

- les préoccupations et les données sur l'exploitation durable et l'environnement;

- la gestion des valeurs non ligneuses et des superficies forestières exploitables en décroissance;

- la planétarisation des échanges commerciaux, la concurrence et l'opinion publique mondiale;

- le sous-financement et l'échéance des ententes fédérales-provinciales;

- la précision des modèles de forêt en vue d'une exploitation durable;

- le manque de données sur les valeurs socioéconomiques des forêts;

- les revendications territoriales et la foresterie sur les terres amérindiennes;

- l'aménagement intensif des forêts mixtes boréales;

- les facteurs économiques de l'industrie secondaire des produits forestiers;

- l'accroissement des besoins d'information à tous les niveaux;

- les nouvelles politiques provinciales sur la foresterie et la protection des forêts;

- l'exploitation de fermes forestières pour la production de bois et de rideaux-abris.

Le financement de la recherche varie considérablement d'une province à l'autre. Les subventions accordées en vertu d'ententes fédérales-provinciales sont toutefois essentielles partout. Les sujets considérés comme sous-financés sont énumérés ci-dessous; ils sont classés en fonction du ratio indiqué dans la colonne de droite, qui représente le nombre de provinces et de territoires ayant désigné le sujet en question comme étant sous-financé sur le nombre de régions l'ayant signalé comme prioritaire.

Gestion des ravageurs et des mauvaises herbes et solutions de rechange aux produits chimiques

Effets de l'aménagement forestier sur

l'environnement 
Connaissances écologiques pour l'aménagement intensif des forêts

Données sur l'accroissement et le rendement des forêts

Gestion et répression des incendies de forêt

Gestion intégrée des ressources et appui à la décision

Inventaire des forêts et classification des sites Méthodes de sylviculture et de récolte et réduction de leurs coûts

Accroissement de la productivité, amélioration des arbres et régénération

Gestion des forêts mixtes
$2 / 4$

$2 / 4$

$1 / 3$

$1 / 5$

$0 / 1$
La recherche effectuée sur les forêts au Canada est considérée comme bien orientée, mais l'application locale des résultats pourrait être améliorée.

\section{Introduction}

Le Conseil consultatif de la recherche forestière du Canada (CCRFC) a compilé ce quatrième survol annuel des priorités de la recherche à l'intention du Conseil canadien des ministres des forêts (CCMF). La compilation fait suite à la proposition avancée en 1987, voulant que chaque province et chaque territoire établissent un organisme consultatif de recherche forestière et qu'un rapport soit publié annuellement sur le classement des sujets de recherche par priorité. Toutes les provinces et tous les territoires sont maintenant dotés d'un organisme fonctionnel, qui joue un rôle primordial dans les survols annuels. La plupart regroupent des représentants des gouvernements, de l'industrie et des universités ainsi que des groupes intéressés par des produits autres que le bois ou par l'environnement et des groupes autochtones. Le conseil consultatif de la recherche forestière de la Colombie-Britannique s'est adjoint un représentant de l'association provinciale des éleveurs de bovins.

Les survols des priorités de recherche sont présentés chaque année aux ministres, en vue de leur réunion automnale, et publiés subséquemment dans le Forestry Chronicle. Ce quatrième rapport est fondé sur les renseignements fournis par les groupes provinciaux au début de 1992.

La compilation des données ne cesse d'évoluer en se simplifiant; les rapports des participants sont aussi de plus en plus concis. Ces derniers répondaient cette année aux questions suivantes:

- Nommer les cinq premières priorités de la recherche forestière dans votre province ou territoire, par ordre décroissant d'importance. La question est en fait de savoir quels travaux de recherche sont nécessaires à l'amélioration de l'aménagement forestier et au maintien durable de tous les avantages de la forêt ?

- Des cinq sujets nommés, indiquer ceux qui sont insuffisamment financés et soutenus.

- Décrire les questions qui se font jour et qui devraient vraisemblablement influer sur les méthodes forestières et, par conséquent, l'ordre des priorités.

- Commenter la qualité et la quantité des travaux de recherche menés dans votre région.

Les réponses ont été envoyées au secrétaire de direction du CCRFC, qui a rédigé un rapport préliminaire et l'a soumis à l'examen d'une réunion commune du CCRFC et des représentants des organismes consultatifs des provinces et territoires, en mai 1992. Le rapport a ensuite été revu avant sa présentation au CCMF.

L'Ontario n'a pu fournir de réponse, puisque l'organisme consultatif est en pleine restructuration. Or, comme l'ordre

Tableau 1. Priorités de recherche, 1992

\begin{tabular}{|c|c|c|c|c|c|c|c|c|c|c|c|}
\hline Sujet & T.-N. & N.-É. & Î.-P.-É. & N.-B. & Qc & Ont. & Man. & Sask. & Alb. & C.-B. & Total \\
\hline $\begin{array}{l}\text { Gestion intégrée des ressources } \\
\text { et appui à la décision }\end{array}$ & 2 & 2 & 4 & 2 & 4 & 1 & 4 & 5 & 2 & 4 & 30 \\
\hline $\begin{array}{l}\text { Gestion des ravageurs et des } \\
\text { mauvaises herbes et solutions de } \\
\text { rechange aux produits chimiques }\end{array}$ & & 3 & 3 & 3 & 2 & 8 & 2 & 1 & & & 22 \\
\hline $\begin{array}{l}\text { Effets de l'aménagement forestier } \\
\text { sur les forêts }\end{array}$ & 3 & 1 & & & & & 5 & 3 & 3 & 5 & 20 \\
\hline $\begin{array}{l}\text { Connaissances écologiques pour } \\
\text { l'aménagement intensif des } \\
\text { forêts }\end{array}$ & 4 & & 5 & & 5 & & & & & 3 & 17 \\
\hline $\begin{array}{l}\text { Données sur la croissance et le } \\
\text { rendement des forêts }\end{array}$ & 5 & & & 5 & & & 3 & & & 1 & 14 \\
\hline $\begin{array}{l}\text { Accroissement de la productivité, } \\
\text { amélioration des arbres et } \\
\text { régénération }\end{array}$ & 1 & 4 & & & & 2 & & & 5 & 2 & 14 \\
\hline $\begin{array}{l}\text { Inventaire des forêts et } \\
\text { classification des sites }\end{array}$ & & & 2 & 4 & & & & 4 & & & 10 \\
\hline $\begin{array}{l}\text { Méthodes de sylviculture et de } \\
\text { récolte et réduction de leurs } \\
\text { coûts }\end{array}$ & & 5 & & 1 & 3 & & & & & & 9 \\
\hline $\begin{array}{l}\text { Gestion et répression des } \\
\text { incendies de forêt }\end{array}$ & & & 1 & & & 4 & 1 & 2 & & & 8 \\
\hline Gestion des forêts mixtes & & & & & & & & & 4 & & 4 \\
\hline
\end{tabular}

Nota - La cote 5 représente la priorité la plus élevée et la cote 1, la priorité la plus faible.

Toutes les priorités à l'exception d'une seule sont considérées comme inadéquatement financées et comme candidates à une aide supplémentaire. 
des priorités n'y change pas tellement d'année en année, ce sont les réponses de l'année dernière qui sont incorporées au rapport de l'année 1992. Tout comme auparavant, les territoires n'ont pas soumis de réponses.

\section{Les priorités de la recherche}

Les renseignements donnés sur les priorités de la recherche ont été mis sous forme de tableau, selon l'origine et le sujet. Puis a été dressée une liste de 10 sujets, signalés parmi les cinq priorités d'un ou de plusieurs organismes consultatifs. Les sujets les plus prioritaires ont obtenu une cote de 5 , ceux qui venaient au deuxième rang ont reçu une cote de 4 et ainsi de suite, pour chaque province et territoire. La liste (tableau 1) est présentée par ordre décroissant des priorités ainsi établies. Il n'y a que quatre sujets dans la colonne réservée à l'Ontario, deux des sujets présentés l'année dernière ayant été combinés sous une même rubrique; les cotes ont été ajustées en conséquence. Aucun des sujets de la liste ne constitue une nouveauté en 1992. Le libellé, le groupement et l'attention portée à chacun ont changé, mais tous ont été abordés par les survols antérieurs.

Les sujets prioritaires sont étroitement liés. Ainsi les deux qui viennent en tête de liste, soit l' 'aménagement intégré des ressources et l'appui à la décision" de même que les «effets de l'aménagement forestier sur l'environnement» sont assez dépendants des «connaissances écologiques pour l'aménagement intensif des forêts".

La recherche sur la transformation du bois, les nouveaux produits, les produits de valeur supérieure, le recyclage et la réduction des besoins énergétiques est considérée comme importante dans le maintien de la position concurrentielle du bois comme matériau et du Canada comme nation commerçante. Il a également été question de la nécessité de produits qui permettraient d'utiliser au mieux les stocks actuels. La recherche sur les produits forestiers n'est toutefois pas l'objectif premier du CCRFC, de sorte que ces sujets n'ont pas été cotés.

Les systèmes modernes de traitement de l'information, l'intelligence artificielle et la biotechnologie ont aussi été jugés importants, mais comme il s'agit d'outils plutôt que de sujets de recherche, ils n'ont pas non plus été cotés.

Les sujets de recherche, présentés par ordre décroissant de priorité, sont discutés dans les sections qui suivent.

\section{Gestion intégrée des ressources et appui à la décision}

La priorité absolue de cette année combine en fait deux sujets présentés l'année dernière, soit «systèmes de gestion intégrée des ressources" et "systèmes d'appui à la décision". La pression qu'exerce le public pour que les forêts soient davantage utilisées à des fins récréatives et de préservation de la faune restreint la superficie utilisable pour la production de bois.

Les progrès dépendent de l'amélioration de l'information. Il faudra en effet améliorer les techniques de prise d'inventaire et d'évaluation de la faune, des débits des cours d'eau et de la valeur récréative des forêts et il faudra mieux connaître la succession des communautés forestières, l'habitat faunique et les réactions des populations aux méthodes d'aménagement forestier. Il faudra enfin des données plus justes sur la croissance et le rendement des forêts et une information plus complète sur les conséquences écologiques des modes de traitement, comme il en est fait mention sous d'autres rubriques.

Pour mieux utiliser l'information connue, il importe de se doter de meilleurs systèmes d'appui aux décisions relatives à l'aménagement forestier ainsi qu'à l'utilisation des terres. Il faudra que soient conçus des modèles et des systèmes d'intelligence artificielle conviviaux, grâce auxquels il sera possible d'intégrer toutes les données obtenues sur les facteurs influençant le choix de l'aménagement forestier et de l'utilisation des terres en plus d'évaluer les compromis nécessaires au choix de la meilleure option. Cela fait ressortir tout de suite l'importance des recherches menées sur deux autres sujets prioritaires, soit les effets de l'aménagement forestier sur l'environnement et la nécessité d'une compréhension plus approfondie des écosystèmes forestiers.

\section{Gestion des ravageurs et des mauvaises herbes et solutions de rechange aux produits chimiques}

Le besoin de meilleures techniques de gestion, y compris l'élimination de la végétation concurrente, vient au deuxième rang des priorités, et la demande de solutions de rechange aux produits chimiques constitue l'exigence la plus considérable. Les solutions aux problèmes de cette nature sont tributaires des progrès accomplis dans les domaines prioritaires connexes, par exemple l'écologie forestière et la gestion intégrée des ressources. Les avantages et les risques inhérents à toutes les solutions de rechange possibles, qu'il s'agisse de l'immobilité ou d'une intervention majeure, doivent faire l'objet d'une évaluation minutieuse. Il importe en outre de trouver de meilleurs moyens d'évaluer les dangers et les dommages pouvant découler d'une invasion de ravageurs.

\section{Effets de l'aménagement forestier sur l'environnement}

Il importe de mieux comprendre l'incidence des méthodes forestières actuelles et à venir sur l'environnement forestier global. Comme il a été mentionné plus haut, il faut dresser l'inventaire de toutes les valeurs forestières et trouver de nouvelles façons de les mettre en état et de les intégrer aux systèmes modernes de classification des sites forestiers, de sorte que leur utilité soit accrue, dans le temps comme dans l'espace. Il sera également essentiel d'exercer une surveillance à long terme pour détecter tout changement et d'adopter de nouvelles méthodes à cette fin, mais aussi de trouver comment neutraliser les effets nocifs de l'aménagement. Il serait bon de commencer par chercher d'autres méthodes de récolte, plus souvent combinées au reboisement que les méthodes actuelles. L'objectif ultime est toutefois de concevoir un système global capable de coordonner toutes les activités, depuis les premières étapes de la planification jusqu'à la production d'un programme d'aménagement des forêts qui tienne compte de toutes les valeurs forestières. Il importera également de donner aux travailleurs une formation qui les sensibilise à la protection de l'environnement et de comprendre comment leur travail peut influer sur l'environnement dans son ensemble. Cet aspect de la question nécessitera toutefois une recherche spécialisée, fondée en partie sur l'apport de la main-d'oeuvre elle-même. 


\section{Connaissances écologiques pour l'aménagement intensif des forêts}

La connaissance approfondie de la dynamique et du fonctionnement des écosystèmes forestiers est un pilier de la gestion globale et durable des ressources forestières. Cette exigence est étroitement liée aux priorités décrites plus haut, qui montrent la nécessité de systèmes de gestion intégrée et d'une évaluation minutieuse des effets, sur l'écosystème forestier, de la chasse, des récoltes, de la pêche, du vent, des ravageurs, du feu et des changements climatiques à l'échelle planétaire.

\section{Données sur la croissance et le rendement des forêts}

La planification de l'exploitation des forêts pour la production de bois repose sur un bon inventaire des forêts et sur des données relatives à la croissance et au rendement des forêts, qui constituent un besoin permanent de bon nombre de régions. Le perfectionnement et le raffinement des modèles forestiers informatiques au fur et à mesure que de nouvelles données seront connues ne peut qu'améliorer la compréhension des liens et clarifier les options offertes en matière d'aménagement.

\section{Accroissement de la productivité, amélioration des arbres et régénération}

Il est possible de répondre à la demande nationale de bois à partir de superficies réduites en trouvant des façons d'accélérer et de rentabiliser la régénération à l'aide de stocks de meilleure qualité, de soins culturaux de peuplements améliorés, d'une meilleure protection contre les ravageurs et les incendies, ainsi que de techniques sylvicoles plus efficaces. Les gestionnaires de territoires auront un choix plus grand d'utilisations débouchant sur des avantages plus diversifiés et plus considérables. De plus grandes superficies pourront être consacrées aux loisirs, à la préservation de la faune, aux réserves fauniques, au pâturage et à d'autres fins. Cette priorité est étroitement associée à d'autres, qu'elle englobe même, en un sens. L'objectif est ici d'améliorer l'application de l'information essentielle produite par la recherche menée sur d'autres sujets prioritaires.

\section{Inventaire des forêts et systèmes de classification des sites}

Les inventaires constituent les données les plus fondamentales de l'aménagement forestier. Comme le champ d'action de l'aménagement s'élargit pour viser toutes les ressources forestières, la demande d'inventaires plus justes et plus accessibles se fait de plus en plus pressante. La conception et l'application de systèmes améliorés de classification des sites en fonction de leur productivité permettra d'étendre la validité des résultats des recherches sans grand risque d'erreur, de sorte que les méthodes d'aménagement qui réussissent dans une région donnée pourront être appliquées avec un certain degré de confiance dans d'autres régions.

\section{Méthodes de sylviculture et de récolte et réduction de leurs coûts}

La rentabilisation des méthodes de sylviculture et de récolte sont prioritaires en partie à cause du climat actuel d'austérité. D'autres facteurs sont la nécessité, mentionnée plus haut, de relier les techniques de récolte et de régénération plus étroitement et celle de concevoir des systèmes sans danger pour l'environnement et durables à long terme. Il importe d'adopter des méthodes de sylviculture et d'élimination des ravageurs plus proches de la dynamique naturelle des forêts et qui perturbent moins les écosystèmes forestiers. Citons entre autres la régénération naturelle plutôt que la plantation, un désherbage manuel efficace plutôt que des herbicides et la diversification de préférence à la monoculture. Ces méthodes se révèlent plus rentables si l'on tient compte de considérations écologiques à long terme.

\section{Gestion et répression des incendies de forêt}

Le feu endommage toujours gravement les forêts canadiennes. Chaque année, plus d'un million d'hectares sont brûlés et les pertes atteignent parfois des chiffres astronomiques. Le réchauffement planétaire pourrait bien aggraver la situation; il faut donc y prêter une attention soigneuse. Les systèmes informatisés d'intégration des données et de gestion des ressources consacrées à la lutte contre l'incendie ont grandement amélioré la capacité de riposte. La recherche continue sur l'amélioration de ces méthodes et l'élargissement de leur champ d'application ne peut que porter ses fruits.

\section{Gestion des forêts mixtes}

La gestion des forêts mixtes illustre bien la variabilité des préoccupations relatives aux forêts sur le territoire canadien. L'Alberta classe le sujet au deuxième rang de ses priorités à cause de ses vastes forêts composées à la fois de peupliers et de coniferres. Le rétablissement des conifères au sein de forêts mixtes, leur protection contre l'incendie et les ravageurs ainsi que l'instauration de conditions propices à leur croissance sont particulièrement préoccupants.

\section{L'évolution des priorités}

Il semble opportun, après quatre survols annuels, d'analyser l'évolution des priorités. Il est impossible de faire une comparaison rigoureuse, puisque la méthode de compilation des données a été modifiée au bout de deux ans. On peut toutefois assigner une cote globale (priorité élevée, moyenne, etc.) pour les deux premières années aux sujets énumérés dans la liste des priorités. Il a fallu cependant prendre quelques libertés pour adapter la classification antérieure des sujets à la liste simplifiée et condensée que nous avons obtenue en demandant à chaque province et à chaque territoire de ne nommer que cinq priorités. Malgré tout, les résultats, bien que non déterminants, montrent certaines tendances (tableau 2).

Il est impossible de discerner quelque mouvement spectaculaire; il est tout de même bon de noter que le premier sujet, "gestion intégrée des ressources et appui à la décision", figure toujours parmi les priorités les plus élevées. Il est aussi significatif de noter que les trois sujets suivants, tous étroitement liés aux préoccupations environnementales, ont grimpé dans l'échelle pour atteindre maintenant les positions 2,3 et 4 . Il s'agit de «lutte contre les ravageurs et les mauvaises herbes et solutions de rechange aux produits chimiques", «effets de l'aménagement forestier sur l'environnement» et «connaissances écologiques pour l'aménagement intensif des forêts». "Méthodes de sylviculture et de récolte et réduction de leurs coûts» est passé d'une priorité faible à une priorité moyenne, tandis que deux autres sujets : «données sur la 
Tableau 2. Évolution des priorités de recherche de 1989 à 1992

\begin{tabular}{|c|c|c|c|c|}
\hline Sujet & $\begin{array}{l}\text { Priorité } \\
89\end{array}$ & $\begin{array}{l}\text { Priorité } \\
90\end{array}$ & $\begin{array}{l}\text { Priorité } \\
91\end{array}$ & $\begin{array}{l}\text { Priorité } \\
92\end{array}$ \\
\hline Gestion intégrée des ressources et appui à la décision & Élevée & Élevée & $\begin{array}{l}\text { Moyenne } \\
\text { à élevée }\end{array}$ & Élevée \\
\hline $\begin{array}{l}\text { Gestion des ravageurs et des mauvaises herbes et solutions } \\
\text { de rechange aux produits chimiques }\end{array}$ & Moyenne & Élevée & Élevée & Élevée \\
\hline Effets de l'aménagement forestier sur l'environnement & Moyenne & $\begin{array}{l}\text { Moyenne } \\
\text { à élevée }\end{array}$ & Élevée & $\begin{array}{l}\text { Moyenne } \\
\text { à élevée }\end{array}$ \\
\hline Connaissances écologiques pour l'aménagement intensif des forêts & Faible & Moyenne & $\begin{array}{l}\text { Moyenne } \\
\text { à élevée }\end{array}$ & $\begin{array}{l}\text { Moyenne } \\
\text { à élevée }\end{array}$ \\
\hline Données sur la croissance et sur le rendement des forêts & Élevée & Élevée & Moyenne & Moyenne \\
\hline $\begin{array}{l}\text { Accroissement de la productivité, amélioration des arbres et } \\
\text { régénération }\end{array}$ & Élevée & Élevée & $\begin{array}{l}\text { Moyenne } \\
\text { à faible }\end{array}$ & Moyenne \\
\hline Inventaire des forêts et classification des sites & Moyenne & Moyenne & Moyenne & Moyenne \\
\hline $\begin{array}{l}\text { Méthodes de sylviculture et de récolte et réduction de leurs } \\
\text { coûts }\end{array}$ & Faible & Moyenne & Moyenne & $\begin{array}{l}\text { Moyenne } \\
\text { à faible }\end{array}$ \\
\hline Gestion et répression des incendies de forêt & Moyenne & Moyenne & Moyenne & $\begin{array}{l}\text { Moyenne } \\
\text { à faible }\end{array}$ \\
\hline Gestion des forêts mixtes & - & - & - & Faible \\
\hline
\end{tabular}

Tableau 3. Facteurs pouvant influer sur les priorités de recherche en 1992

\begin{tabular}{|c|c|c|c|c|c|c|c|c|c|c|c|}
\hline Sujet & T.-N. & N.-É. & Î.-P.-É. & N.-B. & Qc & Ont. & Man. & Sask. & Alb. & C.-B. & Total \\
\hline $\begin{array}{l}\text { Préoccupations et données } \\
\text { sur l'exploitation durable } \\
\text { des forêts et sur } \\
\text { l'environnement }\end{array}$ & 1 & 1 & 1 & & 1 & & 1 & 1 & 1 & 1 & 8 \\
\hline $\begin{array}{l}\text { Gestion des valeurs non } \\
\text { ligneuses et des superficies } \\
\text { forestières exploitables en } \\
\text { décroissance }\end{array}$ & 1 & & 1 & 1 & & & & 1 & 1 & 1 & 6 \\
\hline $\begin{array}{l}\text { Planétarisation des échanges } \\
\text { commerciaux, concurrence } \\
\text { et opinion publique mondiale }\end{array}$ & 1 & 1 & 1 & & 1 & & & & 1 & 1 & 6 \\
\hline $\begin{array}{l}\text { Sous-financement et échéance } \\
\text { des ententes fédérales- } \\
\text { provinciales }\end{array}$ & 1 & 1 & & & & & & & & 1 & 3 \\
\hline $\begin{array}{l}\text { Précision des modèles de forêt } \\
\text { en vue d'une exploitation } \\
\text { durable }\end{array}$ & & 1 & & & & & 1 & & & & 2 \\
\hline $\begin{array}{l}\text { Manque de données sur les valeurs } \\
\text { socio-économiques des forêts }\end{array}$ & & & & & 1 & & 1 & & & & 2 \\
\hline $\begin{array}{l}\text { Revendications territoriales } \\
\text { et foresterie sur les } \\
\text { terres amérindiennes }\end{array}$ & & & & & & & 1 & 1 & & & 2 \\
\hline $\begin{array}{l}\text { Aménagement intensif des forêts } \\
\text { mixtes boréales }\end{array}$ & & & & & & & & 1 & 1 & & 2 \\
\hline $\begin{array}{l}\text { Facteurs économiques de } \\
\text { l'industrie secondaire des } \\
\text { produits forestiers }\end{array}$ & & & & & & & & 1 & & & 1 \\
\hline $\begin{array}{l}\text { Accroissement des besoins } \\
\text { d'information précise à tous } \\
\text { les niveaux }\end{array}$ & & & & & 1 & & & & & & 1 \\
\hline $\begin{array}{l}\text { Nouvelles politiques provinciales } \\
\text { sur la foresterie et la protec- } \\
\text { tion des forêts }\end{array}$ & & & & & 1 & & & & & & 1 \\
\hline $\begin{array}{l}\text { Exploitation de fermes fores- } \\
\text { tières pour la production de } \\
\text { bois et de rideaux-abris }\end{array}$ & & & & & & & & 1 & & & 1 \\
\hline
\end{tabular}


croissance et le rendement des forêts» et «accroissement de la productivité, amélioration des arbres et régénération» ont tendance à descendre l'échelle. La position des autres sujets n'a pas considérablement changé depuis quatre ans.

On a craint plus tôt que les priorités puissent changer plus rapidement que les organismes de recherche ne pouvaient s'y intéresser. Ce ne semble pas avoir été le cas pour les priorités de recherche en foresterie au cours des quatre dernières années.

\section{Facteurs qui peuvent influer sur les priorités de recherche}

Douze facteurs ont été mentionnés comme pouvant modifier la nature des priorités de recherche à venir. Beaucoup ne l'ont toutefois été que par un ou deux participants (tableau 3).

Les préoccupations du public canadien et celles d'autres pays relativement à l'exploitation durable des forêts et à l'environnement ont été mentionnées par tous les participants sauf un. Les priorités soulignées dans le présent rapport reflètent d'ailleurs cette préoccupation. La cote élevée que les participants accordent à la gestion intégrée des ressources, aux effets de l'aménagement forestier sur l'environnement et à la recherche de solutions de rechange aux pesticides chimiques l'illustre bien. L'adéquation aux exigences changeantes de la société du concept de développement durable qui, dans le cas des forêts, exige une planification à très long terme, constitue un défi de taille. Il faut, pour le relever avec succès, intensifier la recherche menée sur le fonctionnement de l'écosystème forestier et trouver de nouvelles méthodes d'aménagement forestier.

La demande mondiale en produits forestiers devrait augmenter encore, sans compter l'ouverture de nouveaux marchés, mais les nouveaux producteurs livreront une concurrence féroce et les marchés existants pourraient bien être restreints. Comme il a été dit plus haut, l'avenir du secteur forestier et de son apport majeur à l'économie canadienne repose sur la découverte de nouveaux produits forestiers et sur l'amélioration de la qualité des produits existants.

\section{Financement}

Comme prévu, le financement varie considérablement d'une région à l'autre, mais les subventions de recherche attribuées en vertu d'ententes fédérales-provinciales ont partout une importance considérable. La liste des sujets de recherche considérés comme sous-financés figure ci-dessous, par ordre décroissant. C'est dire que le premier sujet de la liste est jugé le moins adéquatement financé. Le ratio, à droite, indique le nombre de provinces et de territoires qui voient le sujet sous-financé par rapport au nombre de provinces et de territoires qui l'ont porté sur leur liste de sujets prioritaires. Les deux sujets classés les plus mal financés ont trait à l'environnement.

Lutte contre les ravageurs et les mauvaises herbes et solutions de rechange aux produits chimiques Effets de l'aménagement forestier sur

l'environnement

Connaissances écologiques pour l'aménagement intensif des forêts

Données sur l'accroissement et le rendement des forêts

Gestion et répression des incendies de forêt

Gestion intégrée des ressources et appui à la décision

Inventaire des forêts et classification des sites

Méthodes de sylviculture et de récolte et réduction de leurs coûts

Accroissement de la productivité, amélioration des arbres et régénération

Gestion des forêts mixtes

Comme tous les sujets à l'exception d'un seul sont considérés comme ne bénéficiant pas de subventions de recherche adéquates, nous pouvons interpréter cette liste, combinée à celle des priorités, comme une revendication de subsides supplémentaires.

\section{À-propos de la recherche forestière}

Bon nombre des participants ayant répondu à cette question insistent sur l'aspect financement. Leur évaluation devait se fonder sur deux critères difficiles d'application : l'adéquation entre les besoins et la recherche ainsi que la qualité des travaux. Les personnes qui ont risqué un commentaire pensent que la recherche forestière qui s'effectue dans leur région est de bonne qualité et qu'elle correspond assez aux besoins perçus, mais qu'il faudrait améliorer l'application des résultats. 